\title{
El Estado Actual de la Investigación Sobre la Discriminación Sexual
}

\author{
The State or the Art in Research on Sexual Discrimination
}

\author{
José Toro-Alfonso \\ Universidad de Puerto Rico
}

(Rec: 5 de abril de 2012 / Acep: 5 de mayo de 2012)

\begin{abstract}
Resumen
En la medida en que el mundo se ha globalizado y las fronteras parecen ser cada vez más difusas, pareciera que algunos sectores de la sociedad tienen la necesidad de acentuar las diferencias y poner distancia de esas diferencias. Esto parece observarse en los niveles de exclusión y rechazo que reciben las personas que pertenecen a las comunidades de lesbianas, gays, bisexuales y transgéneros. Desde una perspectiva de las prácticas basadas en evidencia y de los derechos humanos, este artículo presenta el estado actual de las investigaciones sobre la discriminación sexual. La homofobia individual e institucionalizada sirve como base central de la discriminación. Se discute la transgresión del género como elemento fundamental para justificar el odio y la discriminación que puede llevar al homicidio. Se plantea la necesidad de que desde la psicología se desarrollen estándares éticos para las intervenciones en estas comunidades.
\end{abstract}

Palabras clave: Discriminación sexual, homofobia, diversidad sexual y psicología

\begin{abstract}
As the world is more globalized and frontiers are constantly more diffused, some sectors of society have the need to underline differences and establish distance from these differences. This seems to be observed in the levels of social exclusion and rejection that members of the gay, lesbian, bisexual and transgender communities receive. From a perspective of evidence based practices and human rights, this article present the current state of research on sexual discrimination. Individual and institutional homophobia is presented as a key element for discrimination. It is discussed that gender transgression is also a fundamental element for the justification of hate that might lead to homicide. The need for psychology to develop ethical standards for interventions in these communities is presented.
\end{abstract}

Keywords: Sexual discrimination, homophobia, sexual diversity and psychology

\section{Introducción}

En la medida en que el mundo se ha globalizado y las fronteras parecen ser cada vez más difusas, pareciera que algunos sectores de la sociedad tienen la necesidad de acentuar las diferencias y poner distancia de esas diferencias. Estas situaciones se hacen evidentes en términos religiosos, de etnicidad y origen nacional. En Latinoamérica se incluyen también los sectores indígenas y las ideas políticas.

La insistencia en las diferencias entre los grupos y dar privilegio a un grupo por encima de los demás, lo que se ha denominado "orientación social dominante" (Pratto, Sidanius, Stallworth \& Malle, 1994), da origen a elaborar estereotipos y juicios a priori. Esta es principalmente la base del prejuicio intergrupal. La base de la discriminación suele encontrarse casi siempre en los estereotipos, que son creencias, ideas y sentimientos negativos o positivos hacia ciertas personas pertenecientes a un grupo determinado. Cuando se realiza una valoración negativa de un grupo en base al estereotipo, el resultado es el prejuicio. Cuando los prejuicios llevan a una persona a actuar de un modo determinado respecto al grupo o individuo prejuzgado, el resultado es la discriminación.

\section{Las actitudes, el prejuicio y la orientación sexual}

Fueron Adorno, Frenkel-Brunswik, Levinson \& Sanford (1950) quienes realizaron los primeros estudios sobre prejuicio, estableciendo básicamente que el autoritarismo de derecha era la base principal del prejuicio. Aunque muchos de sus planteamientos fueron criticados (Altemeyer, 1981; Martin, 2001) hoy sabemos que la investigación reciente sostiene que el autoritarismo de derecha se correlaciona con toda una variedad de prejuicios (Altemeyer, 1996; Duckitt \& Farre, 1994; McFarland, Ageyev \& Abalakina, 1993).

Correspondencia: La correspondencia relacionada con este artículo debe ser enviada a Dr. José Toro-Alfonso. Facultad de Ciencias Sociales, Departamento de Psicología, Universidad de Puerto Rico, San Juan PO Box 23345, Puerto Rico. E-mail: jose.toro4@upr.edu 
Las actitudes negativas hacia la homosexualidad y el lesbianismo se han estudiado desde muchas perspectivas, especialmente intentando identificar cómo se desarrollan y cómo se pueden facilitar intervenciones para disminuir el prejuicio y el rechazo social. En un intento por caracterizar a las personas con prejuicio hacia los gays y las lesbianas, Herek $(1984,1994)$ indica que aquellas que manifiestan más actitudes negativas hacia la homosexualidad poseen actitudes tradicionales sobre los roles de género, tienen más amistades que manifiestan actitudes negativas, son mayores en edad, tienen menos educación formal y son conservadoras en asuntos religiosos. Otros investigadores han mencionado que los niveles de prejuicio y rechazo hacia la homosexualidad están relacionados con los modelos de atribución. Estos estudios señalan que las actitudes se relacionan con la percepción de que la persona tiene o no el control sobre su homosexualidad (Sakalli, 2002).

Los estudios sobre el prejuicio contra las minorías sexuales han coincidido sobre cuáles son las características principales de las personas que discriminan. Se destacan en la mayoría de los estudios, las ideas políticas conservadoras, la asistencia regular al culto religioso, actitudes negativas hacia la mujer y una fuerte adherencia al modelo hegemónico de la masculinidad y la división de roles sexuales.

Sin embargo, hay autores que señalan que el prejuicio se hace cada vez más sofisticado y difícil de identificar, dando paso a niveles sutiles de prejuicio y discriminación. Nos indican Quiles del Castillo, Betancor-Rodríguez, Rodríguez -Torres, Rodríguez-Pérez \& Coello-Martel (2003): “La presencia de fuertes presiones normativas en favor de la igualdad y la tolerancia, en lugar de eliminar este prejuicio parece haberlo hecho más sutil y sofisticado" (p. 198). Todo parece indicar que el prejuicio homosexual tiene muchos parecidos con el autoritarismo y con el prejuicio racial, aun con todas sus diferencias. Concluyen Quiles et al. (2003) que:

"Nuestros resultados confirman la idea de que la homofobia o el prejuicio hacia homosexuales presenta características similares al prejuicio racial. Probablemente porque las condiciones sociales en que se producen ambos fenómenos son las mismas" (p. 204).

\section{La homofobia}

La homofobia se manifiesta en una respuesta afectiva y en actitudes negativas basadas en mitos y estereotipos acerca de las relaciones entre personas del mismo sexo (Snively, Kreurger, Stretch, Watt \& Chadha, 2004). La homofobia, como el racismo y la misoginia representan obstáculos mayores para el pleno disfrute de los derechos humanos que toda persona ciudadana debe tener garantizados. De hecho algunos investigadores sobre el tema han planteado que en la sociedad occidental en donde se condena el racismo y el antisemitismo, y en donde la misoginia ha perdido legitimidad, la homofobia permanece quizás como el último prejuicio aceptado socialmente (Fone, 2000).
Aun en jóvenes universitarios podemos encontrar altos niveles de homofobia, lo que pudiera contradecir la percepción de que la juventud educada es más tolerante a la diferencia y a la diversidad sexual (Toro-Alfonso \& Varas-Díaz, 2004).

Mercado-Mondragón (2009) señala que existe un nivel de homofobia personal que resulta en la creencia de que las personas homosexuales y lesbianas merecen el odio bajo el supuesto de que no pueden controlar sus deseos, que son mayormente anormales. Por otro lado, existe la homofobia interpersonal que surge cuando el prejuicio personal se transforma en acciones discriminatorias. Así mismo, la homofobia institucional permite que se ejerza presión contra el sector homosexual en las instituciones educativas, religiosas, empresariales y profesionales. Concluye Mercado-Mondragón que:

"Para combatir y erradicar la homofobia se requieren acciones urgentes que incidan en un cambio de patrón cultural que ha establecido a la heterosexualidad como la única opción válida para el ejercicio del amor y de la sexualidad. Si la sociedad sigue sin reconocer a la homosexualidad como otra opción, los homicidas homofóbicos continuarán argumentando que con el asesinato de homosexuales liberan a la sociedad de entes depravados que no tienen derecho a vivir" (p. 152).

En la vida cotidiana los homosexuales y las lesbianas se enfrentan a diferentes formas de prejuicio, además de que existen pocas organizaciones que atiendan los problemas específicos de esta población y no hay reconocimiento institucional y legal de los distintos arreglos familiares entre individuos del mismo sexo. Las formas más evidentes de violencia van desde los insultos verbales y las agresiones físicas, hasta formas extremas como los asesinatos (OrtizHernández \& García-Torres, 2005).

Los crímenes de odio por homofobia no son asesinatos entre homosexuales. Muchas veces el asesino no es homosexual y hay víctimas que tampoco lo son. Son asesinatos provocados por el odio que los grupos de poder han ido construyendo en la sociedad a lo largo de siglos contra toda forma de deseo y práctica sexual no convencional, y no solamente contra los homosexuales y la homosexualidad (Rodríguez, 2010).

Algunos investigadores (Parrot, Peterson, Vincent \& Bakeman, 2008) plantean que existe una relación entre el coraje que media la respuesta de prejuicio sexual y agresión hacia los hombres homosexuales, lo que implica la necesidad de examinar los factores relacionados con la manifestación del coraje. Los resultados de sus investigaciones han demostrado que la adherencia a los modelos hegemónicos de la masculinidad se relaciona con la agresión hacia la diferencia sexual. Aquellos hombres que se inscriben en el modelo dominante de una masculinidad heterosexual, agresiva y dominante, tienden a manifestar conductas dentro del continuo de la homofobia. Esto va desde el chiste 
impersonal, el rechazo, los comentarios, la agresión y la posibilidad del homicidio.

De acuerdo con Levin y McDevitt (1993), los crímenes de odio surgen desde el contexto social y no deben ser tratados como incidentes aislados cometidos por un grupo de personas mentalmente perturbadas. La historia del desarrollo de las legislaciones en los diferentes países relacionados a los crímenes de odio, ha confrontado precisamente el nivel de homofobia institucional que se reconoce en los Estados. La dificultad para definir la intencionalidad y el pensamiento de odio de parte del agresor hace difícil establecer diferencias entre los crímenes en general y los crímenes de odio.

Sin embargo, aunque el prejuicio no es más moralmente reprobable que cualquier otra motivación para el crimen, existe evidencia de que son diferentes. La psicología y la criminología establecen claramente que los crímenes de odio pueden causar mayor daño a la víctima y a la comunidad en comparación con otros crímenes. El nivel de violencia utilizado, la brutalidad del evento y los significados asociados a los crímenes de odio dan cuenta de que se intenta impactar más allá de la víctima particular (Sullaway, 2004).

\section{La discriminación sexual}

Los supuestos que sustentan la discriminación son muchas veces no considerados claramente y algunos investigadores plantean que es difícil reconocer que participamos de estas actitudes. Sevilla-González (2007) plantea que:

"La actitud ni siquiera es percibida por la mayoría de los sujetos como prácticas discriminatorias porque se consideran estas actitudes como aceptables y válidas, la respuesta es compartida así como los prejuicios y valoraciones" (p. 64).

La realidad es que la violencia y la discriminación contra personas de las comunidades LGBT es parte de un discurso normalizado que es aceptado socialmente y que no parece provocar grandes disonancias cognitivas en las personas. Así se tolera la violencia y se justifica en ocasiones por una "moral" no explicitada.

El silencio -en ocasiones, cómplice- permite el espacio para toda una serie de crímenes de odio que se producen en América Latina y el Caribe. Aunque es probable que no se reporten claramente todos los casos, se han informado casos muy reconocidos en Chile, Argentina (Comunidad Homosexual Argentina, 2009), Perú (Movimiento Homosexual de Lima, 2012), Colombia (Velandia, 2008), México (Brito \& Bastida, 2009), Brasil (Mongrovejo, s.f.), Guatemala (Colectivo Amigos contra el Sida, 2010), Boliva, Nicaragua, Honduras (G Noticias, 2011), Venezuela (Colectivo de Lesbianas Feministas, 2009), República Dominicana (El Nacional, 2011) y Puerto Rico (ToroAlfonso, 2007). En los Estados Unidos, Herek (2009) ha realizado estudios sobre el nivel de estigma y discriminación con muestras probabilística.

Estos eventos no incluyen la proliferación de intervenciones psicológicas sin base empírica que se utilizan para "curar" la homosexualidad, en ocasiones con medidas violentas. Las terapias de curación para homosexuales han sido fuertemente criticadas y consideradas como violentas por todas las organizaciones profesionales de salud mental en los Estados Unidos y en América Latina (Santiago \& Toro-Alfonso, 2010).

\section{La inversión de género como el límite para la aceptación}

En muchos de los estudios sobre prejuicio y discriminación (Ortíz-Hernández, 2004; Toro-Alfonso, 2009) se hace evidente que la adhesión a los cánones tradicionales de la representación social del género es un requisito importante para la inclusión social en América Latina. Los estereotipos que señalan que todos los hombres gay parecen mujeres y que todas las lesbianas parecen hombres, descansa en la base de la percepción que tiene la sociedad sobre las comunidades LGBT.

No es sorprendente que una sociedad como la nuestra, que tradicionalmente ha sido tan rígida en el manejo de los roles sexuales y que exige concordancia, tenga grandes dificultades en todo lo que le parezca que invierte el género. Ya sea porque lo ven como un "problema hormonal"; "un problema genético":

"Somos testigos de cómo el género es una de las categorías reguladoras de mayor importancia en nuestra sociedad. A través de éste, se ha ejercido control sobre las personas desde instancias históricas que preceden nuestra era, tomando el dictamen de la anatomía biológica como punto de partida para constituirnos en hombres o en mujeres" (Rodríguez-Madera \& Toro-Alfonso, 2002, p. 64).

Los asuntos de género parecen ser el tema final para la tolerancia. Muchas personas manifiestan dificultades en el manejo de las personas transgénero, incluyendo hombres gay "afeminados" o mujeres que "parecen hombres". Evidentemente relacionado a los mitos y estereotipos, las risas, miradas, chistes y comentarios que se observan frente a personas que rompen con el esquema tradicional del género.

Los comentarios sobre cómo se identifica a una persona gay o lesbiana están también vinculados al estereotipo del género. Muchas personas piensan que pueden reconocer a los homosexuales y lesbianas precisamente porque muestran rasgos confusos sobre el género o se les observa una clara inversión del género (Toro-Alfonso, 2006).

Tradicionalmente provoca risas y chistes el hecho de que un hombre-que socialmente posee el potencial para el poder y la masculinidad dominante- se represente socialmente 
con la imagen femenina. Los medios de comunicación televisivos están llenos de la presencia casi patética de la imagen de un pobre hombre homosexual que se muestra como frágil, afeminado y con pretensiones histéricas. Para la sociedad, provoca risas que precisamente un hombre sea capaz de adoptar semejante postura..., lo femenino, que pareciera tan desvalorizado y rechazado en nuestra sociedad.

También la mirada del género le asigna a los hombres gay características positivas: son buenos amigos, tienen buenos gustos, se puede contar con ellos, saben escuchar, visten muy bien... todas estas características socialmente asignadas a lo femenino. La imagen transgénero también se observa en esta línea. En ocasiones percibida como la exageración de lo femenino, la persona transexual remite a toda la confusión que provocan las imágenes de travestis y transexuales famosas y conocidas por la televisión (hiper mujer, seductora, provocadora y sobre todo sin pelos en la lengua).

Por otro lado, la imagen de la lesbiana es totalmente lo contrario. La lesbiana se representa socialmente como la persona arribista. La mujer que no reconoce su posición social y se atreve a pretender la masculinidad, privilegio permitido sólo a los hombres biológicos. La lesbiana no da gracia... provoca temor. No es la primera vez que escuchamos comentarios y advertencias sobre la brutalidad -casi masculina- de la lesbiana. Se les presenta como "pasionales", que "se enamoran de todas las mujeres" y presentan grandes escenas de celos y de agresión.

\section{Para una plena ciudadanía}

Es imposible garantizar los derechos civiles de un sector social dentro del contexto de la exclusión y la violación de los derechos humanos. Evidentemente, dentro de este marco los derechos sexuales son derechos humanos. La marginación, la pobreza, la falta de acceso a servicios de salud y protección y la falta de reconocimiento de la variedad de identidades (situaciones que llevan en ocasiones hasta el asesinato), son violaciones de los derechos a la plena ciudadanía de los hombres homosexuales, lesbianas, bisexuales y transgéneros (Díaz, Ayala, Bein, Henne \& Marín, 2001).

Las violaciones a la plena ciudadanía parecen ser constantes y generalizadas en nuestros países, y la bruta realidad es que estas situaciones conmueven poco al resto de la comunidad. Todos los seres humanos tienen derecho a la vida plena, y la diversidad en orientaciones e identidades sexuales no debe representar un obstáculo para la felicidad. Se hace inaplazable que se establezcan límites a las intervenciones policíacas y a la actitud permisiva y tolerante que estimula a sectores de las mayorías a agredir y marginar a los homosexuales. El gobierno y sus instituciones hacen un débil servicio a la comunidad cuando no reconocen la responsabilidad que les corresponde.

Es importante reconocer que los homosexuales enfrentan discriminación social, cultural y a veces legal y económica, debido a su comportamiento sexual, con efectos nefastos para su salud mental y el libre disfrute de la vida (Carleton, 1999; Mays \& Cochran, 2001). Desarrollar y fortalecer políticas públicas que protejan los derechos de este sector de la comunidad significa permitir el acceso de las poblaciones vulnerables a escenarios laborales dignos, a servicios de salud preventiva y a la libre expresión de su sexualidad. Se hace imperante que el gobierno y la sociedad civil establezcan planes de desarrollo y solidaridad para prestar atención a uno de los sectores más vulnerables de nuestra sociedad.

\section{Discusión}

La discusión y el debate sobre la homosexualidad continúa siendo un punto de referencia importante en momentos en que el espacio social y político se reduce cada vez más. A pesar de los grandes adelantos en países desarrollados en donde existe legislación que protege a las personas de las comunidades LGBT, existen países en donde el péndulo se mueve hacia modelos conservadores y de mayor control social. En los países en desarrollo existe un vacío de reconocimiento de la diversidad de la sexualidad y mucho más existe un espíritu de eliminación de la diferencia. Aun en países supuestamente desarrollados, como los Estados Unidos, no podemos negar que el intento de control de la derecha religiosa va tomando auge con mucha facilidad.

La imposición del orden de la mayoría, que niega la diferencia y no reconoce la complejidad de las relaciones humanas puede ser tan cruel como capaz de tolerar la violencia y los crímenes de odio. Desde la psicología nos corresponde ofrecer dirección y evidencia científica del daño que provoca la exclusión y nos corresponde desarrollar guías claras a los profesionales y a toda la comunidad de los elementos éticos que deben seguir nuestras intervenciones. Algunas asociaciones de psicología han dado el primer paso para establecer recomendaciones y estándares para el trabajo psicológico con las poblaciones LGBT (Asociación de Psicología de Puerto Rico, 2008).

Nos corresponde a todos y todas tender puentes y fortalecer una sociedad que se distinga por la tolerancia y la celebración de la diversidad. De eso se trata la diversidad humana.

\section{Referencias}

Adorno, T. W., Frenkel-Brunswik, E., Levinson, D. J., \& Sanford, R. N. (1950). The authoritarian personality. New York: Harper, \& Brothers. Altemeyer, B. (1981). Right-wing authoritarianism. Winnipeg, CA: University of Manitoba Press.

Altemeyer, B. (1996). The authoritarian specter. Cambridge, MA: Harvard University Press.

Asociación de Psicología de Puerto Rico (2008). Estándares para el trabajo e intervención en comunidades LGBT. Recuperado de: http:// www.asppr.net/pdf/LGBT.pdf

Brito, A., \& Bastida L. (2009). Crímenes de odio por homofobia: México 1995-2008. México, DF: Letra S, Sida, Cultura y Vida Cotidiana A.C. 
Carleton, F. (1999). Contested identity: The law's construction of gay and lesbian subjects. En L. Pardie, \& T. Luchetta (Eds.), The construction of attitudes toward lesbian and gay men (pp. 19-37). Nueva York, NY: The Harworth Press.

Colectivo Amigos Contra el SIDA(2010). Crimenes de odio en Guatemala. Recuperado de: http://www.amigoscontraelsida.net/docs/crimenesdeodioenguatemala.pdf

Colectivo de Lesbianas Feministas (2009). Venezuela: No más crímenes de odio por orientación sexual e identidad de género. Recuperado de: http://www.insurrectasypunto.org/index.php?option=com_conte nt\&view=article\&id=1341:venezuela-no-mas-crimenes-de-odio-pororientacion-sexual-e-identidad-de-genero\&catid=3:notas \&Itemid=3

Comunidad Homosexual de Argentina (CHA) (2009). Informe sobre lesbofobia, transfobia, homofobia, bi fobia en Latinoamérica y el Caribe. Recuperado de: http://www.cha.org.ar/articulo.php?art=364\&cat=5

Díaz, R., Ayala, G., Bein, E., Henne, J., \& Marín, B. (2001). The impact of homophobia, poverty, and racism on the mental health of gay and bisexual Latino men: Findings from 3 U.S. cities. American Journal of Public Health, 91, 927-932.

Duckitt, J., \& Farre, B. (1994). Right-wing authoritarianism and political intolerance among whites in the future majority-rule South Africa. Journal of Social Psychology, 134, 735-741.

El Nacional (2011, octubre). Hallan homosexual asesinado. Periódico digital. Recuperado de: http://www.asylumlaw.org/docs/sexualminorities/DomRepublicElNacional100511.pdf

Fone, B. (2000). Homophobia: A history. New York: Picador USA, A Metropolitan Book, Henry Holt and Company.

G Noticias (2011). La homofobia en Latinoamérica en números. Recuperado de: http://www.radarg.com/index.php/noticias/?p=6496

Herek, G. (1984). Beyond homophobia: A social psychological perspective on attitudes toward lesbians and gay men. Journal of Homosexuality, $10,2-17$.

Herek, G. (1994). Assessing heterosexuals' attitudes toward lesbians and gay men. A review of empirical research with the ATLG Scale. En B. Greene \& G. Herek (Eds.), Lesbian and gay psychology (pp. 206-228). Thousand Oaks, CA: Sage.

Herek, G. M. (2009). Hate crimes and stigma-related experiences among sexual minority adults in the United States: Prevalence estimates from a national probability sample. Journal of Interpersonal Violence, 24, 54-74.

Levin, J., \& McDevitt, J. (1993). Hate crimes: The rising tide of bigotry and bloodshed, NY: Plenum Press.

Martin, J. L. (2001). The authoritarian personality, 50 years later: What lessons are there for political psychology? Political Psychology, 22, 1-26.

Mays, V., \& Cochran, S. (2001). Mental health correlates of perceived discrimination among lesbian, gay, and bisexual adults in the United States. American Journal of Public Health, 91, 1869-1876.

McFarland, S., Ageyev, V., \& Abalakina, M. (1993). The authoritarian personality in the United States and the former Soviet Union: Comparative studies. In W. F. Stone, G. Lederer, \& R. Christie (Eds.), Strength and weakness: The authoritarian personality today (pp. 199-225). New York: Springer-Verlag.

Mercado-Mondragón, J. (2009). Intolerancia a la diversidad sexual y crímenes por homofobia: Un análisis sociológico. Sociológica, 24, 123-156.

Movimiento Homosexual de Lima (MHOL) (2012). El camarada "Artemio" reconoce asesinato de homosexuales. Recuperado de: http:// www.mhol.org.pe/
Mogrovejo, N. (s.f.). Transfobia y homofobia en Latinoamérica. Recuperado de: http://agmagazine.com.ar/index.php?IdNot=2438

Ortiz-Hernández, L. (2004). La opresión de minorías sexuales desde la inequidad de género. Política y Cultura, 22, 131-182.

Ortiz-Hernández, L., \& García-Torres, M.I. (2005). Efectos de la violencia y la discriminación en la salud mental de bisexuales, lesbianas y homosexuales en la Ciudad de México. Cadernos de Saúde Pública, 21,913-925.

Parrot, D.J., Peterson, J.L., Vincent, W., \& Bakeman, R. (2008). Correlates of anger in response to gay men: Effects of male gender roles beliefs, sexual prejudice, and masculine gender role strain. Psychology of Men and Masculinity, 8, 167-178.

Pratto, F., Sidanius, J., Stallworth, L. M., \& Malle, B. F. (1994). Social dominance orientation: A personality variable predicting social and political attitudes. Journal of Personality and Social Psychology, 67, 741-763.

Quiles del Castillo, M.N., Betancor-Rodríguez, V., Rodríguez-Torres, R. Rodríguez -Pérez, A., \& Coello-Martel, E. (2003). La medida de la homofobia manifiesta y sutil. Psicothema, 15, 197-204.

Rodríguez, E. (2010). Un crimen de odio por homofobia en Ciudad de Juárez. El Cotidiano, 164, 61-67.

Rodríguez-Madera, S., \& Toro-Alfonso, J. (2002). Ser o no ser: La transgresión del género como objeto de estudio de la psicología. Avances en Psicología Clínica Latinoamericana, 20, 63-78.

Santiago, M., \& Toro-Alfonso, J. (2010). La cura que no cura: Una mirada crítica a las terapias reparativas de la homosexualidad y el lesbianismo. Revista Salud \& Sociedad, 1, 136-144.

Sakalli, N. (2002). Application of the attribution-value model of prejudice to homosexuality. Journal of Social Psychology, 142, 264-271.

Snively, C. A., Krueger, L., Stretch, J. J., Watt, J. W., \& Chadha, J. (2004). Understanding homophobia: Preparing for practice realities in urban and rural settings. Journal of Gay and Lesbian Social Services, 17, 59-81.

Sullaway, M. (2004). Psychological perspectives on hate crime laws. Psychology, Public Policy, and Law, 10, 250-292.

Sevilla -González, M. (2007). El discurso homofóbico como expresión de la discriminación sexual. En G. Soberón, \& D. Feinholz (Eds.), Homofobia y Salud (pp. 63-76). México, DF: Secretaría de Salud.

Toro-Alfonso, J., \& Varas-Díaz, N. (2004). Los otros: Prejuicio y distancia social hacia hombres gay y lesbianas en una muestra de estudiantes de nivel universitario. Revista Internacional de Psicología Clínica y de la Salud, 4, 537-551.

Toro-Alfonso, J. (2006). El estudio de las homosexualidades: Revisión, retos éticos y metodológicos. Revista de Ciencias Sociales, 14, 78-97.

Toro-Alfonso, J. (2007). Por la vía de la exclusión: Homofobia y ciudadanía en Puerto Rico. San Juan, PR: Comisión de Derechos Civiles de Puerto Rico.

Toro-Alfonso, J. (2009). La inversión del género como límite a la aceptación de empleados y empleadas de gobierno en Puerto Rico hacia la comunidad gay. Cuadernos de la Revista Cayey, 3, 141-158.

Velandia, M. (2008). Crimenes de odio a minorías sexuales en Colombia. Recuperado de: http://agmagazine.com.ar/index.php?IdNot=2438 